\title{
An Optimized Design of a Parallel Robot for Gait Training
}

\author{
Marco Maddalena $^{1}$, Mozafar Saadat ${ }^{1}$, Alireza Rastegarpanah ${ }^{1}$ and Rui C. V. Loureiro ${ }^{2}$
}

\begin{abstract}
The guidelines for enhancing robot-assisted training for post-stroke survivors head towards increasing exercise realism and variability; in particular lower limb rehabilitation needs the patient to feel challenged to adapt his locomotion and dynamic balance capabilities to different virtual ground scenarios. This paper proposes a design for a robot whose endeffector acts as a footplate to be in permanent contact with the user's foot during practice: the structure is such that it enables the user's foot to rotate around three axis, differently from what is currently available in the research for gait training; the parallel kinematic structure and the dimensional synthesis allow a suitable range of motion and aim at limiting device mass, footprint and reaction forces on the actuators when rendering virtual ground. The employed methodology has been validated using ground reaction forces data relative to stroke survivors.
\end{abstract}

\section{INTRODUCTION}

According to World Health Organisation, 15 million people suffer stroke every year globally [1] and a majority of the stroke survivors suffer critical gait disorders. The recovery of gait function requires the patient to undergo a task-specific therapy: conventional manual training responds to this request for therapy but the rehabilitation process is slow, expensive and dependent on therapists' skills.

In the last two decades different robotic devices have been developed for providing assistance during the gait therapy [2] and they can be divided into two main categories according to the working principle: exoskeleton plus treadmill systems (e.g.. Lokomat, AutoAmbulator, LOPES, ALEX) and endeffector robots (e.g. G-EO-systems, Haptic Walker, Gait Trainer, Gait Master). Some of these devices have had clinical application, leading to the reduction in required labour and time and the growth of motor exercise intensity and volume; nonetheless the therapeutic outcome of currently available robotic gait trainers is still questionable [3].

End-effector technology can be seen as the starting point for a performance enhancement due to the increased range of motion provided to the pelvis, which is not restricted by an exoskeleton. The end-effector based robotic gait rehabilitation systems developed so far provide the user with two footplates which can simulate different kinds of virtual ground like slopes and stairs but the motion is limited to the sagittal plane.

Conversely, it has been suggested [4] that therapy effectiveness is strictly related to exercise parameters variability;

\footnotetext{
${ }^{1}$ Department of Mechanical Engineering, School of Engineering, University of Birmingham, B15 2TT Birmingham, Email: mxm715@bham.ac.uk, m.saadat@bham.ac.uk, a_r_adrex@yahoo.com

${ }^{2}$ Aspire Centre for Rehabilitation Engineering and Assistive Technology, University College London, Royal National Orthopaedic Hospital, Stanmore, HA7 4LP London, Email: r.loureiro@ucl.ac.uk
}

the increase in motor learning is due to presenting each action as a problem to be solved, rather than a bare succession of muscle activity patterns to be memorized and then iterated, so that it is the task, not the simple movement, that has to be repeated during training.

In particular introducing exercise variability during poststroke rehabilitation therapy is believed to lead to:

- therapy compliance enhancement: ability to change has therapeutic benefit as the brain has the potential to remap the area affected by injury/stroke in response to training of skilled tasks and hence allow motor recovery [5];

- outcome retention: performance improvement is not limited to the training session itself, but can be acknowledged also in subsequent trials [6];

- outcome generalization: the skills acquired can be transfered to activities of daily living (ADL) [4];

- the overcoming of the so called "plateau": chronic stroke patients can achieve important improvement even over one year after the occurence [7].

Applying these principles to gait rehabilitation therapy, it is plausible that integrating three axis rotation into footplate motion capability could be an important step towards higher performance training, since allowing the user to simulate walking on ground whose surface is inclined on different planes provides a substantial postural control demand, increasing the challenge of the training [8]. It must be remarked furthermore that such a stimulating effect due to surface variability is already exploited by devices for balance training [9], [10], where multiple-degree of freedom perturbations include roll, pitch and yaw axis of rotation.

The need to merge the high force required for supporting the user's body weight during stance phase and the possibility of rotating the platform around all the axes presents an obstacle from a mechanical realization viewpoint. The proposed system is a parallel robot that aims at overcoming such an obstacle and providing the following benefits, when employed as a footplate for locomotion training:

- Three degrees of freedom for platform rotation, so surfaces with slopes around different axes can be rendered;

- The whole mechanism is under the user's feet providing the user with safety and confidence while improving the possibility by the therapist to interact with the patient;

- The area employed by the device is limited, so that the user can simulate walking without collision and with a proper distance between the feet;

- Reduced mass for an improved horizontal dynamic performance, so as to ensure a high exercise repetition 
rate and accordingly suitable therapy intensity.

Section II reports the features the robot shall fulfill and the limitations of current robotic solutions to be overcome by the new device. Section III describes the structure and the kinematics of the proposed robot. Section IV presents the methodology for the optimisation of the robot dimensions. Finally, section V provides demonstration of the applicability of the proposed design.

\section{ApProACH TO THE PROBlem}

The robot will be permanently connected to the user's feet during the training and during the stance phase it shall emulate ground reaction forces.

\section{A. Ground reaction forces during stance phase}

With the aim to allow the use of the gait training device by a wider population, the general conditions on the interaction between the ground and the foot during contact shall be extrapolated from analysis of step trials. Ground reaction forces acting on a foot during walking can be measured by force plates [11] which give the centre of pressure as the weighted average of the location of all downward forces: during stance the center of pressure moves forward, starting from the heel towards the metatarsal head and the toe, usually with two distinct vertical force peaks.

The sum of the vertical ground reaction forces exerted by the feet can exceed body weight, in fact, as the center of mass of the body accelerates and decelerates vertically, the inertial effect has to be added to or subtracted; however the vertical ground reaction force on a single foot is usually under $120 \%$ of body weight.

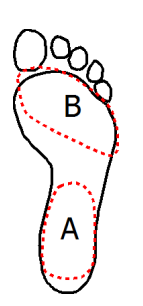

(a)

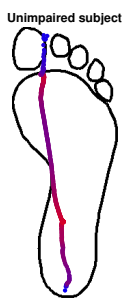

(b)

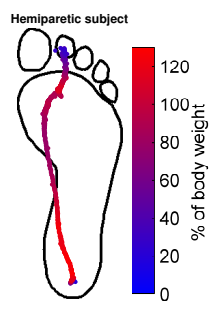

(c)
Fig. 1. (a) zones of the foot plant where the centre of pressure is likely to be located during vertical support force peaks, corresponding to a location between the heel and the midfoot (zone A) and a location on the metatarsal head in the forefoot (zone B); (b-c) position of the centre of pressure along the foot during stance phase represented with the vertical ground reaction force; the length and the width of the foot have been normalised.

The horizontal force acting along the longitudinal direction initially decelarates the body center of mass, while after midstance it becomes propulsive for the push off phase. The absolute value of this force is smaller than vertical ground reaction force, generally reaching around $20 \%$ of body weight. Also the horizontal force in the transversal direction is limited to less than $10 \%$ of body weight, as it is required only for balance purpose.

Data relative to healthy and post-stroke individuals has been collected [12] in order to compare unimpaired subjects

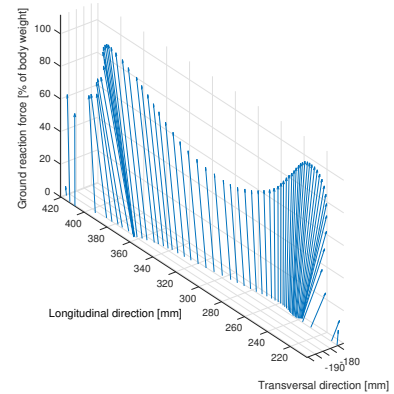

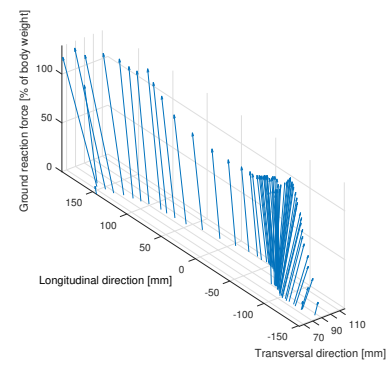

Fig. 2. Butterfly diagrams of ground reaction forces during stance: (a) unimpaired subject and (b) hemiparetic subject.

and stroke survivors (figure 1 and 2): the gait parameters of a number of volunteers were recorded and analysed in the gait lab of West Midland Rehabilitation Centre (WMRC), Birmingham. Participants completed informed consent form to take part in this study which had acquired the relevant ethical approval. The gait lab was equipped with two force plates (with frequency of $1000 \mathrm{~Hz}$ ).

Figure $1 \mathrm{~b}$ shows the centre of pressure relative to a typical healthy subject: here the force peaks can be identified respectively close to the heel and on the metatarsal head. Figure 1c reports the stance phase of the partially paralysed foot of a typical hemiparetic subject and an uncertain behaviour can be observed; the difference can be better recognized using the butterfly diagram (figure 2): the first peak force almost disappears while the centre of pressure shifts quickly to the forefoot where it persists longer in a shaky manner. Nonetheless, it can be inferred that the peaks of force on the forefoot have a comparable magnitude and take place in the same area (zone B of figure 1a).

\section{B. Current mechanical solutions for robot-assisted gait re- habilitation}

The robots suitable to render variable ground that have been proposed so far in the research are based on footplates. In order to cope with workspace demands, most of devices are structured so that the motion along longitudinal direction of walking is implemented with an actuated linear slide, while vertical motion and eventually rotation are given by additional mechanisms mounted on the sliding base platform.

An example of such a system is the locomotion interface GaitMaster5 [13] provided with two footpads that can move only horizontally and vertically without any rotation, but dorsal and plantar flexion are left free beacuse of a particular device used to fix the foot joints to the footpad. Other two examples are given by "Haptic Walker" [14] and "G-EO system" [15]: they are simulators able to perform walking trajectories including rotation, although limited to the sagittal plane for plantar- and dorsiflexion during the steps.

\section{DESCRIPTION OF THE PROPOSED PARALLEL ROBOT}

The proposed device aims at providing an alternative to what has been presented in the previous subsection: it will 
be installed on a base platform sliding along the longitudinal direction of walking, replacing the mechanism for footplate vertical motion and rotation.

\section{A. Structural synthesis}

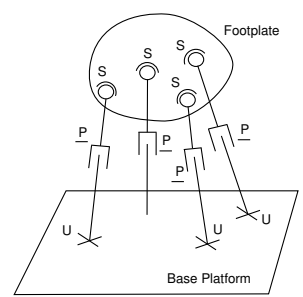

Fig. 3. The kinematic structure of the proposed robot. U stands for universal joint, $\mathrm{P}$ for prismatic joint and $\mathrm{S}$ for spherical joint.

The choice of a parallel kinematic structure is driven by the high payload over mass ratio, the relative compactness and the possibility to confine the entire mechanism under the footplate. In order to allow vertical motion and three axis rotation the robot shall perform 4 degrees of freedom: the 3 UPS/PS structure (figure 3) proposed in [16] for ankle training can be made suitable for gait training.

The chosen structure can be seen as a particular case of the translation/rotation uncoupled robot presented in [17]; the vertical PS link controls the vertical position of the footplate and can be sufficiently sturdy in order to support both the first vertical force peak and the horizontal forces exerted by the user on the footplate: the horizontal forces are acting off-axis on the link but, as brought up in section II, their magnitude is much smaller than vertical force.

The UPS links instead, bearing only axial force, collaborate to rotate the footplate and support user's body weight when, in the last part of the stance phase, the centre of pressure is transfered to the forefoot.

\section{B. Kinematics}
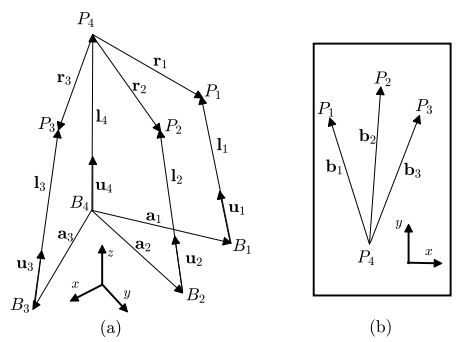

Fig. 4. Definition of the points and the vectors for the robot kinematics: (a) the whole mechanism; (b) the footplate.

Any footplate is going to be the specular image of the other, so hereafter we take into consideration the footplate for the right foot. The $\mathrm{z}$-axis is taken as the vertical direction, the $y$-axis is taken as the longitudinal direction of walking and the $\mathrm{x}$-axis is taken as the transversal direction. The points on the base are marked as $B_{1}, B_{2}, B_{3}, B_{4}$ and the points on the end-effector platform are marked as $P_{1}, P_{2}$,
$P_{3}, P_{4}$ where the indexes 1,2 and 3 refer to the UPS links assigned to rotation and the index 4 to the vertical PS link. The constant vectors $\mathbf{a}_{i}$ connect the points $B_{i}$ to $B_{4}$ on the base (figure $4 \mathrm{a}$ ), while the vectors $\mathbf{b}_{i}$, constant in the end-effector footplate frame, connect the points $P_{i}$ to $P_{4}$ (figure $4 \mathrm{~b}$ ). Defining as $\mathbf{R}$ the rotation matrix of the footplate, the variable vectors $\mathbf{r}_{i}$ connect the points $P_{i}$ to $P_{4}$ on the end-effector footplate, so we have that $\mathbf{r}_{i}=\mathbf{R b}_{i}$. Given the footplate yaw angle $\gamma$, the footplate pitch angle $\alpha$ and the footplate roll angle $\beta$, the rotation matrix can be written as $\mathbf{R}=\mathbf{R}_{z}(\gamma) \mathbf{R}_{x}(\alpha) \mathbf{R}_{y}(\beta)$; if the vertical position of the point $P_{4}$ is marked as $z$ it is possible to define the end-effector pose as $\mathbf{x}=\left[\begin{array}{cccc}z & \alpha & \beta & \gamma\end{array}\right]^{\mathrm{T}}$.

\section{Kinetostatics}

The velocity of the point $P_{i}$ on the the moving platform is $\boldsymbol{v}_{P_{i}}=\left[\begin{array}{ccc}0 & 0 & \dot{z}\end{array}\right]^{\mathrm{T}}+\boldsymbol{\omega} \times \mathbf{r}_{i}$ while for the vertical actuator we have $\boldsymbol{v}_{P_{4}}=\left[\begin{array}{ccc}0 & 0 & \dot{z}\end{array}\right]^{\mathrm{T}}$. Considering that the joint variable derivative is such that $\dot{q}_{i}=\boldsymbol{v}_{P_{i}} \cdot \mathbf{u}_{i}$, defining the vector of translational and angular velocities[ $\left[\begin{array}{ll}\dot{z} & \boldsymbol{\omega}\end{array}\right]^{\mathrm{T}}$ we have the relationship $\dot{\boldsymbol{q}}=\mathbf{J}^{-1}\left[\begin{array}{ll}\dot{z} & \boldsymbol{\omega}\end{array}\right]^{\mathrm{T}}$, where the inverse Jacobian matrix $\mathbf{J}^{-1}$ is:

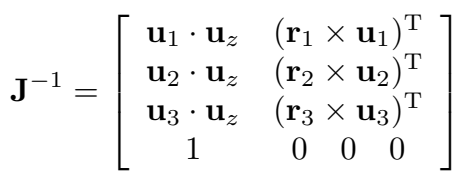

The resultant wrench applied on the point $P_{4}$ is indicated as $\boldsymbol{h}=\left[\begin{array}{cc}f_{z} & \boldsymbol{M}\end{array}\right]^{\mathrm{T}}$ with vertical force $f_{z}$ and torque $M$; if we define the vector of actuator forces $f=$ $\left[\begin{array}{llll}f_{1} & f_{2} & f_{3} & f_{4}\end{array}\right]^{\mathrm{T}}$ we have, for the kinetostatic principle, that the wrench can be obtained as $\boldsymbol{h}=\mathbf{J}^{-\mathrm{T}} \boldsymbol{f}$.

Defining the rotation inverse jacobian submatrix:

$$
\mathbf{J}_{R}^{-1}=\left[\begin{array}{c}
\left(\mathbf{r}_{1} \times \mathbf{u}_{1}\right)^{\mathrm{T}} \\
\left(\mathbf{r}_{2} \times \mathbf{u}_{2}\right)^{\mathrm{T}} \\
\left(\mathbf{r}_{3} \times \mathbf{u}_{3}\right)^{\mathrm{T}}
\end{array}\right]
$$

we have that the resultant vertical force is obtained by the first row of $\mathbf{J}^{-\mathrm{T}}$, that is $f_{z}=\left(\mathbf{u}_{1} \cdot \mathbf{u}_{z}\right) f_{1}+\left(\mathbf{u}_{2} \cdot \mathbf{u}_{z}\right) f_{2}+$ $\left(\mathbf{u}_{3} \cdot \mathbf{u}_{z}\right) f_{3}+f_{4}$, while the resultant torque is

$$
\boldsymbol{M}=\mathbf{J}_{R}^{-\mathrm{T}}\left[\begin{array}{lll}
f_{1} & f_{2} & f_{3}
\end{array}\right]^{\mathrm{T}}=\mathbf{J}_{R}^{-\mathrm{T}} \boldsymbol{f}_{R}
$$

which depends only on the forces on the UPS links because of the diagonal form of $\mathbf{J}^{-\mathrm{T}}$. Accordingly, if the submatrix $\mathbf{J}_{R}^{-1}$ is invertible, we have that $\boldsymbol{f}_{R}=\left[\begin{array}{lll}f_{1} & f_{2} & f_{3}\end{array}\right]^{\mathrm{T}}=$ $\left(\mathbf{J}_{R}^{-\mathrm{T}}\right)^{-1} \boldsymbol{M}$. Moreover we can observe that $\mathbf{J}^{-1}$ is singular if and only if $\mathbf{J}_{R}^{-1}$ is singular.

\section{DimENSIONAL SYNTHESIS}

The aim of the dimensional synthesis is to optimize the structure presented in section III with respect to the requirements outlined in section II. The location of the end point of PS link (point $P_{4}$ in figure 4b) is established under the user's heel. Joints positioning relatively to the UPS links remains as an optimization variable: the parameters being 
taken for the optimization are the planar coordinates of $\mathbf{a}_{1}, \mathbf{a}_{2}, \mathbf{a}_{3}, \mathbf{b}_{1}, \mathbf{b}_{2}, \mathbf{b}_{3}$, so the total number of parameters is 12 .

The design objective of a parallel robot in many cases is achieving isotropy, a property such that the robot can perform velocities and forces in the same way independently from direction. In the present case, conversely, the task is noticeably anisotropic, since there exists a particular direction for the torque to be exerted and the singular values of the jacobian submatrix $\sigma\left(\mathbf{J}_{\mathrm{R}}^{-\mathrm{T}}\right)$ will differ accordingly.

\section{A. Joints position constraint}

The size of the base plate and the footplate shall be limited for reducing device footprint. The length and the width of the platforms are indicated in figure 5, where the gray area marks the available locations for universal joints on the base plate and spherical joints on the footplate.
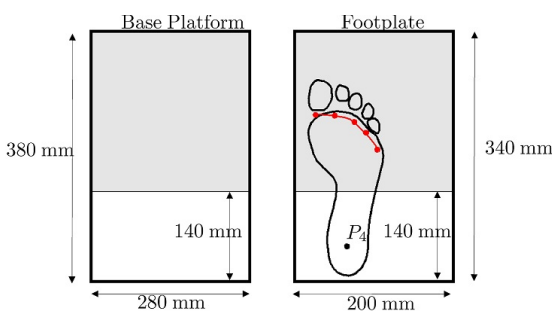

Fig. 5. Dimensions of Base Platform and Footplate.

\section{B. Reference workspace}

Although the set of reachable poses could be further extended, the analysis for optimization has been limited in the following way:

$$
\begin{aligned}
& 420 \mathrm{~mm}<z<570 \mathrm{~mm} \text { Vertical displacement } \\
& -20^{\circ}<\alpha<20^{\circ} \quad \text { Pitch angle } \\
& -20^{\circ}<\beta<20^{\circ} \quad \text { Roll angle } \\
& -20^{\circ}<\gamma<20^{\circ} \quad \text { Yaw angle }
\end{aligned}
$$

The vertical displacement choice is given by the typical rest length and stroke of a linear actuator that could be used for the PS vertical link, while the rotation range is compatible with the one achieved by devices for balance rehabilitation [9]. The pitch angle range has been taken assuming that the foot is fixed to the footpad but, similarly to the GaitMaster5, the user is free to lift the heel. This feature is critical as it allows the user to properly apply force during the push-off phase.

\section{Force conditions}

It is expected that the maximum magnitude of requested torque takes place during the vertical force peak on the forefoot as exposed in section II, so the critical zone where the centre of pressure is going to be is marked as a red arch in figure 5. This zone has been estimated using the anthropometric data about the $95^{\text {th }}$ percentile of British population regarding foot length and breadth [18]. Such data has been exploited for body weight as well, estimating the vertical and horizontal forces that occur during the peak: in the following analysis the extreme ground reaction force is picked equal to $[-100 \mathrm{~N} \quad 300 \mathrm{~N} \quad 1400 \mathrm{~N}]$, so as to take into account people up to $100 \mathrm{Kg}$ with a good safety margin.

\section{Multiobjective function}

Workspace $W$ is the set of reachable points in the endeffector pose space among the ones reported in 4. Some points can't be reached because the UPS link actuators have a limited stroke which has been taken equal to the vertical one $(420-570 \mathrm{~mm})$. Moreover the links of the robots are constrained to lie within a limited volume and this might lead to interference between them at some point of the workspace: this eventuality is checked in a way similar to the one described in [19], but including two different diameters for the link rather than one (the details of the method are omitted for brevity). The variable $W_{p}$ indicates the ratio between $W$ and the reference workspace: it quantifies the capability of the robot to emulate sloped ground at different heights.

Another aim is to accomodate a sufficiently large part of the population in terms of weight and stature, while limiting the mass of the actuators of the UPS links. In order to limit such mass, it is necessary to minimise the maximum static force $f_{\max }$ the actuators have to support during the stance phase in any pose of the workspace $W$, referring to the cases exposed in the previous subsection: the dots on the red arch in figure 5 have been taken as samples for calculating $f_{\max }$.

The demands on the torque capability of the device induce the singular values $\sigma\left(\mathbf{J}_{R}^{-1}\right)$ to be large, but this could impact kinematic transmission performance: in fact recalling that $\left[\begin{array}{ccc}\dot{q}_{1} & \dot{q}_{2} & \dot{q}_{3}\end{array}\right]^{\mathrm{T}}=\mathbf{J}_{R}^{-1} \boldsymbol{\omega}$ it would be beneficial that, given a desired angular velocity, the magnitude of the joint velocities were not excessive.

In order to balance the force and motion range, a local performance index for parameters $\mathbf{a}_{1}, \mathbf{a}_{2}, \mathbf{a}_{3}, \mathbf{b}_{1}, \mathbf{b}_{2}, \mathbf{b}_{3}$ computed at the pose $\mathbf{x}$ is defined as the largest singular value $\sigma_{\max }\left(\mathbf{J}_{R}^{-1}(\mathbf{x})\right)$; accordingly the global performance index to be minimised is defined as follows:

$$
\mathrm{K}_{\mathrm{G}}\left(\mathbf{a}_{1}, \mathbf{a}_{2}, \mathbf{a}_{3}, \mathbf{b}_{1}, \mathbf{b}_{2}, \mathbf{b}_{3}\right)=\frac{\int_{W} \sigma_{\max }\left(\mathbf{J}_{R}^{-1}(\mathbf{x})\right) d \mathbf{x}}{\int_{W} d \mathbf{x}}
$$

As it is not possible to compute symbolically the integral, an estimation of the index shall be calculated by sampling the value of the integrand in some sample poses of the workspace and averaging the result.

Finally, the multiobjective function is taken equal to the vector $\left[\begin{array}{lll}-W_{P} & f_{\max } & K_{G}\end{array}\right]$, where the first term has the minus sign because we want to maximise the portion of reachable workspace.

\section{E. Optimization results}

Since the influence of link interference on the reachable workspace might cause the function $-W_{p}$ to have many local minima, the Multiobjective Genetic Algorithm of MATLAB ${ }^{\circledR}$ Optimization Toolbox has been employed and after 


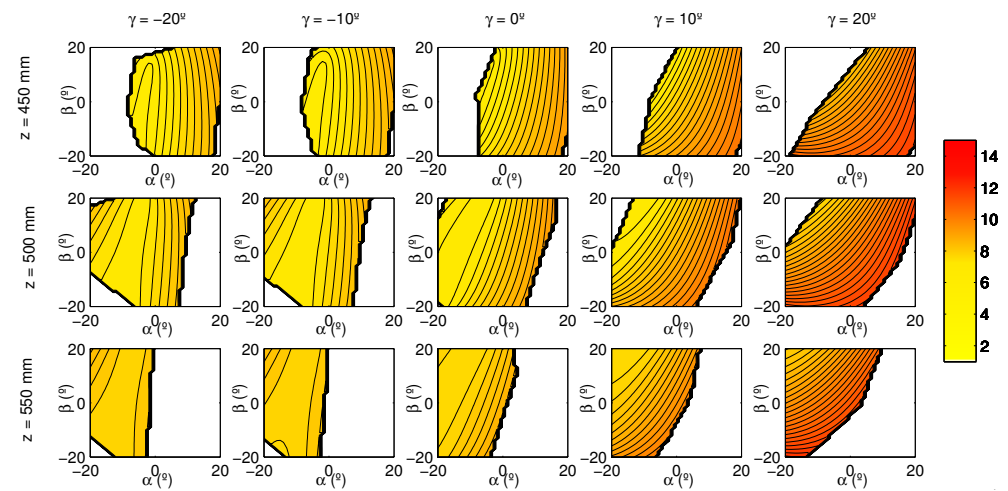

Fig. 6. Local conditioning index $k_{L}$ throughout the reachable workspace.

211 iterations there was convergence to the Pareto front described in figure 7 .
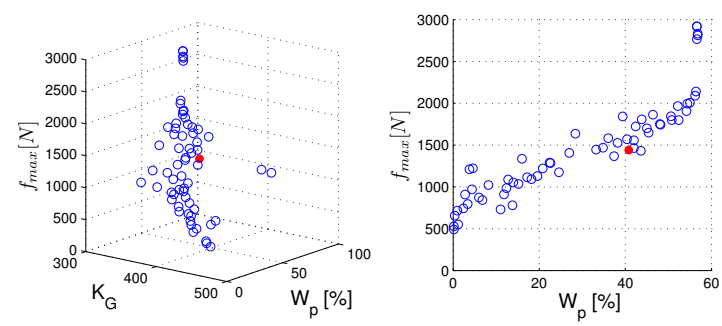

Fig. 7. Pareto front relative to the multiobjective optimization. On the right the projection on the $W_{p}-f_{\max }$ plane.

The optimal parameters of the chosen solution (marked in red on the Pareto front) are reported in table I: it is a trade-off between the objectives described in the previous section, in fact the reachable workspace portion is $40.8 \%$ of the total, while $f_{\max }$ is equal to $1439.1 \mathrm{~N}$, compatible with the maximum static force of lightweight commercial linear actuators. Also the value of $K_{G}$ is a compromise, being equal to 396.5 .

\begin{tabular}{|c|c|c|}
\hline $\mathbf{a}_{1}$ & $\mathbf{a}_{2}$ & $\mathbf{a}_{3}$ \\
\hline \hline$\left[\begin{array}{c}37.9 \\
192.9\end{array}\right]$ & {$\left[\begin{array}{c}4.73 \\
277.3\end{array}\right]$} & {$\left[\begin{array}{l}186.5 \\
239.8\end{array}\right]$} \\
\hline $\mathbf{b}_{1}$ & $\mathbf{b}_{2}$ & $\mathbf{b}_{3}$ \\
\hline \hline$\left[\begin{array}{c}69.9 \\
196.4\end{array}\right]$ & {$\left[\begin{array}{c}-10 \\
235.5\end{array}\right]$} & {$\left[\begin{array}{c}66.3 \\
261.8\end{array}\right]$} \\
\hline
\end{tabular}

TABLE I

OPTIMAL DIMENSIONS IN MILLIMETERS

\section{CONSIDERATIONS ON THE PROPOSED DESIGN}

In this section the proposed kinematic structure is assessed. One feature a parallel robot is requested to fulfill is being singularity-free in the interested workspace: distance from singularity can be rated by evaluating the local conditioning index $k_{L}=\sigma_{\max }\left(\mathbf{J}_{R}^{-1}\right) / \sigma_{\min }\left(\mathbf{J}_{R}^{-1}\right)$, as in figure
6. As the task is anisotropic, we could expect the relatively high value of the conditioning index; however its variability is limited throughout the reachable workspace and it keeps away from singularity, being constantly smaller than 15 .

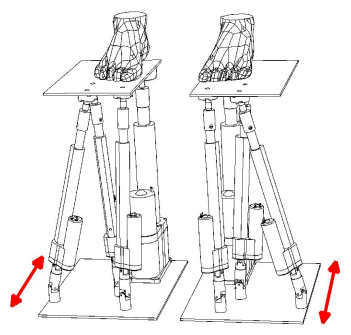

Fig. 8. Rendering of the optimised configuration of the gait training system: the red arrows indicate the longitudinal direction of motion that will be made possible by two actuated linear guide rails.

Although some poses are not reachable beacuse of the mentioned constraints on actuators' stroke, the workspace can be deemed satisfying for the purpose.

The articular forces reacting to load throughout stance phase have been simulated using the ground reaction force data from a stroke survivor (depicted in figure $2 b$ ): this pressure distribution pattern presents typical abnormalities of pathological gait that can be recognised in hemiparetic individuals and provides an example of the situation the robotic device shall face during practice. It should be remarked that the acquisition of such data was carried out with a flat force plate, and in the simulation it was supposed that the centre of pressure would follow the reference frame of the footplate, while the force remained referred to the baseplate frame.

The results relative to different surface orientation are shown in figure 9: it is evident that the PS vertical link is engaged (articular force $f_{4}$ ) in the first stage of the stance phase when the body weight is unloaded on the hindfoot; as the weight is shifted towards the forefoot the UPS links support more and more load (articular forces $f_{1}, f_{2}, \bar{f}_{3}$ ) until the second force peak, while the PS vertical link balances the resultant of the vertical forces.

The unsteady behaviour of the paretic limb causes the articular forces to have an irregular progress, nonetheless 

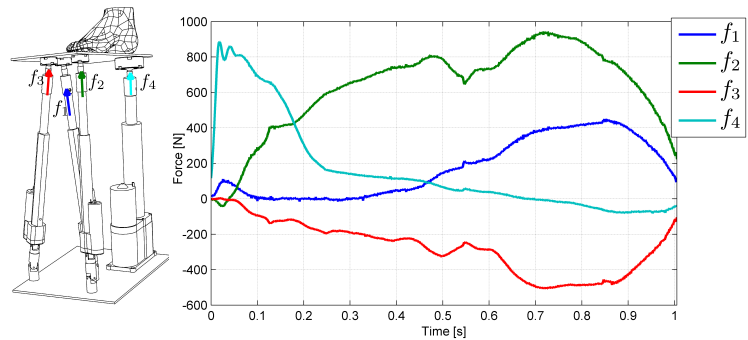

(a)
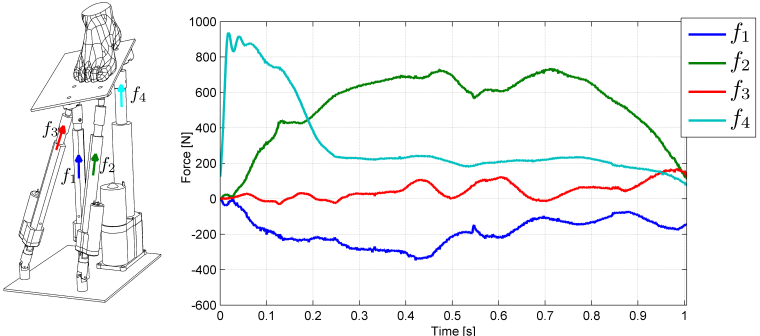

(b)

Fig. 9. Rendering and simulation of the proposed robot: the articular forces of the linear actuators are evaluated in different poses when : (a) $z=$ $490 \mathrm{~mm}, \alpha=10^{\circ}, \beta=-8^{\circ}, \gamma=-10^{\circ}$; (b) $z=510 \mathrm{~mm}, \alpha=-15^{\circ}, \beta=12^{\circ}, \gamma=15^{\circ}$.

the UPS links articular forces in different poses are limited, confirming the appropriateness of the design. The value of force $f_{4}$ can become negative in the final stage of the stance for some poses: this is due to the fact that the UPS link actuators have to withstand also the torque relative to horizontal forces.

\section{Vi. Conclusions}

The paper has presented a process that has led to the kinetostatic optimization of a parallel kinematic mechanism: the obtained structure features three axis rotation with vertical translation, while limiting platform size and articular forces.

These attributes make the device potentially suitable to be used for integrating surface variability during gait training, enhancing what can be currently found in research and following the established guidelines to achieve higher performance therapy for individuals suffering from neurological disorders. The data relative to real patients has been used to simulate the capability of the device to match the ground reaction forces during stance phase, validating the employed methodology.

Future work will comprise devising an arrangement of the overall system for gait training: in particular foreseeing the integration of a longitudinal guide rail and of a connection system between the foot and the platform for practicing walking; moreover user's safety will have to be enquired along with the development of suitable protocols for gait therapy which follow clinical findings on recovery process.

\section{ACKNOWLEDGMENTS}

The authors would like to thank the West Midlands Rehabilitation Centre (WMRC), part of Birmingham Community Healthcare NHS Foundation Trust, for providing gait measurement laboratory support, and Applied Computing and Engineering Ltd. (AC\&E) for their sponsorship of this project.

\section{REFERENCES}

1] World health organization "the atlas of heart disease an stroke (2016)". http://www who.int/cardiovascular_diseases/resources/ atlas/en/. Accessed: 2017-01-08.

[2] P.-Y. Cheng and P.-Y. Lai, "Comparison of exoskeleton robots an end-effector robots on training methods and gait biomechanics," in International Conference on Intelligent Robotics and Applications. Springer, 2013, pp. 258-266.
[3] B. H. Dobkin and P. W. Duncan, "Should body weight-supported treadmill training and robotic-assistive steppers for locomotor training trot back to the starting gate?" Neurorehabilitation and neural repair, vol. 26, no. 4, pp. 308-317, 2012.

[4] J. W. Krakauer, "Motor learning: its relevance to stroke recovery and neurorehabilitation," Current opinion in neurology, vol. 19, no. 1, pp. 84-90, 2006.

[5] A. O. Andrade, A. A. Pereira, S. Walter, R. Almeida, R. Loureiro, D. Compagna, and P. J. Kyberd, "Bridging the gap between robotic technology and health care," Biomedical Signal Processing and Control, vol. 10, pp. 65-78, 2014

[6] T. G. Hornby, J. L. Moore, L. Lovell, and E. J. Roth, "Influence of skill and exercise training parameters on locomotor recovery during stroke rehabilitation," Current Opinion in Neurology, vol. 29, no. 6 , pp. 677-683, 2016

[7] B. H. Dobkin, "Neurorehabilitation interventions to move beyond a plateau in motor outcomes," Current Opinion in Neurology, vol. 29, no. 6 , pp. $675-676,2016$

[8] J. J. Eng and P.-F. Tang, "Gait training strategies to optimize walking ability in people with stroke: a synthesis of the evidence," Expert review of neurotherapeutics, vol. 7, no. 10, pp. 1417-1436, 2007.

[9] F. Patanè and P. Cappa, "A 3-dof parallel robot with spherical motion for the rehabilitation and evaluation of balance performance," IEEE Transactions on Neural Systems and Rehabilitation Engineering, vol. 19 , no. 2 , pp. $157-166,2011$.

[10] A. Lees, J. Vanrenterghem, G. Barton, and M. Lake, "Kinematic response characteristics of the caren moving platform system for use in posture and balance research," Medical engineering \& physics, vol. 29 , no. 5, pp. 629-635, 2007.

[11] T. Marasović, M. Cecić, and V. Zanchi, "Analysis and interpretation of ground reaction forces in normal gait," WSEAS transactions on systems, vol. 8, no. 9, pp. 1105-14, 2009.

[12] A. Rastegarpanah and M. Saadat, "Lower limb rehabilitation using patient data," Applied Bionics and Biomechanics, vol. 2016, 2016.

[13] H. Yano, S. Tamefusa, N. Tanaka, H. Saito, and H. Iwata, "Interactive gait rehabilitation system with a locomotion interface for training patients to climb stairs," Presence, vol. 21, no. 1, pp. 16-30, 2012.

[14] H. Schmidt, S. Hesse, R. Bernhardt, and J. Krüger, "Hapticwalker-a novel haptic foot device," ACM Transactions on Applied Perception (TAP), vol. 2, no. 2, pp. 166-180, 2005.

[15] S. Hesse, A. Waldner, and C. Tomelleri, "Innovative gait robot for the repetitive practice of floor walking and stair climbing up and down in stroke patients," Journal of neuroengineering and rehabilitation, vol. 7 , no. 1 , p. 1,2010

[16] J. S. Dai, T. Zhao, and C. Nester, "Sprained ankle physiotherapy based mechanism synthesis and stiffness analysis of a robotic rehabilitation device," Autonomous Robots, vol. 16, no. 2, pp. 207-218, 2004.

[17] C. Innocenti and V. Parenti-Castelli, "Direct kinematics of the 6-4 fully parallel manipulator with position and orientation uncoupled," in Robotic Systems. Springer, 1992, pp. 3-10.

[18] D. Aler, "Metric handbook planning and design."

[19] O. Masory and J. Wang, "Workspace evaluation of stewart platforms," Advanced robotics, vol. 9, no. 4, pp. 443-461, 1994. 